\title{
CORONARY ARTERY ANEURYSM
}

\section{BY}

\section{GILBERT FORBES, M.D., B.Sc., F.R.C.S.Ed. F.R.F.P.S. Reader in Charge \\ AND}

ANDREW BRADLEY, M.A., M.B., B.S., LL.B. Lecturer

Department of Forensic Medicine, University of Sheffield

Aneurysms of the coronary arteries are rare. A modest total of 70 cases have been reported between 1812 and 1957 (Packard and Wechsler, 1929 ; Scott, 1948 ; Crocker et al., 1957). The condition has been attributed to congenital defect, syphilis, atherosclerosis, rheumatic fever, polyarteritis nodosa, and mycotic embolic phenomena, but many cases have remained unclassified and of unknown aetiology.

\section{Case Report}

This case came to necropsy at the instance of H.M. Coroner for the County Borough of Rotherham, arising out of the death of a boy of 9 . He appeared to be a healthy boy until November 26, 1958, when he began to have occasional headaches and intermittent abdominal pain with vomiting; he also had two attacks of fainting. His appetite was poor and he was sleeping badly. When he went to bed on December 3,1958, he kept complaining of upper abdominal pain, then collapsed and died.

\section{Post-mortem Findings}

The body was that of a well-developed boy, $4 \mathrm{ft}$. 3 in. $(129 \mathrm{~cm}$.) in height. The main findings related to the heart, which weighed $184 \mathrm{~g}$. (average for the age is $115 \mathrm{~g}$.). There was no gross congenital defect of the heart or great vessels, and the valves were healthy. The left ventricle was dilated. The ostium of the left coronary artery was slightly thickened, though its diameter appeared to be within normal limits. The ostium opened immediately into an aneurysm of the

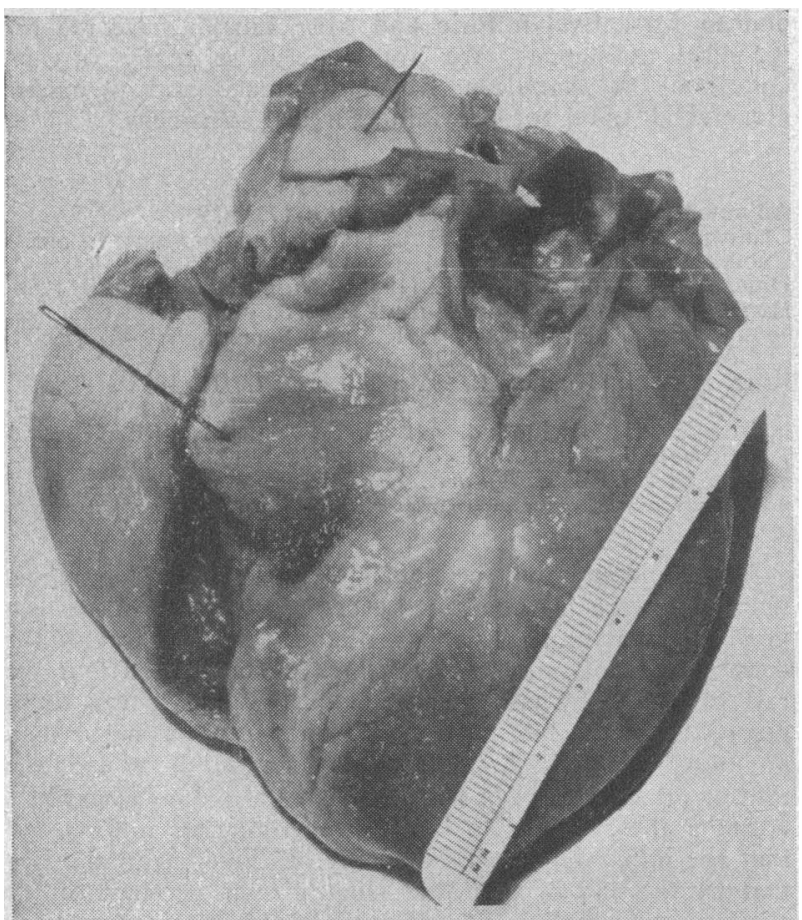

F10. 1.-Heart, showing coronary artery aneurysm opened from the front. The white marker is in the coronary ostium. left coronary artery, filled with laminated blood clot which was friable and loosely adherent to the wall of the artery (Fig. 1). The whole of the main trunk, the first $2 \mathrm{~cm}$. of the anterior descending branch, and the first $0.5 \mathrm{~cm}$. of the circumflex branch were affected by this aneurysmal dilatation. The diameter of the main trunk and descending branch at the site of the aneurysm was generally $1.5 \mathrm{~cm}$., that of the circumflex branch $0.7 \mathrm{~cm}$.

What appeared to be the beginning of the descending and circumflex branches was marked in each case by prominent thickened rings projecting into the lumen of the sac of the aneurysm. The rings met tangentially in the acute angle between the two branches. The descending branch of the left coronary artery was blocked for some distance beyond the aneurysm by red friable clot. The right coronary artery

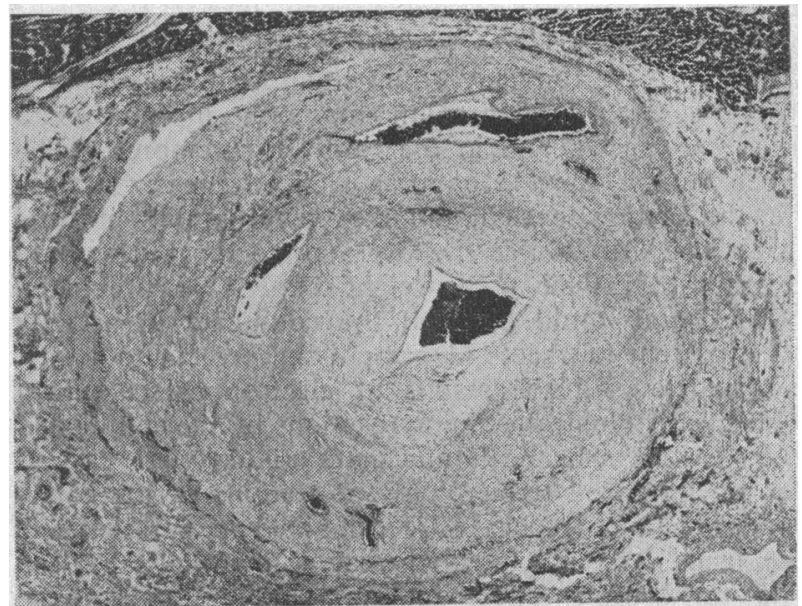

FIG. 2.-Descending branch, left coronary artery, just beyond the aneurysm. (Masson's trichrome and Verhoeff. $\times$ c. 28.)

appeared somewhat narrow. Apart from the aneurysm, the coronary arteries seemed to be healthy; in particular, there was no tortuosity and no atheroma. The muscle of the left ventricle was tough and fibrous, and there was a prominent area of scarring of the interventricular septum measuring 4 by $2 \mathrm{~cm}$. The lungs were very oedematous, there were 6. oz. $(170 \mathrm{ml}$.) of clear fluid in each pleural sac, and an excess of fluid in the pericardium. There was general congestion of the abdominal organs, the spleen being much enlarged. Apart from the features attributable to the condition of the heart, there were no abnormal appearances. In particular, the blood-vessels of the organs other than the heart seemed normal.

\section{Histology}

The wall of the main trunk of the left coronary artery and of those portions of the descending and transverse branches which were the seat of the aneurysm showed gross disorganization of the intima and media. The intima was greatly thickened, and, to describe it from the inside outwards, consisted of, first, the remains of the thrombus, next a latticework of condensed fibrin, then a latticework of comparatively acellular collagen, and finally cellular fibromuscular tissue in the outermost part of the intima. Randomly scattered at various depths in the intima were some amorphous calcareous deposits. While this was the general pattern, here and there the intima had become vascularized. The muscle of the media was in general much thinned out, it was vascularized, and there was a good deal of fibrous tissue replacement. Nothing was to be seen either in the remnants of the thrombus or in the wall of the aneurysm to indicate the presence of any inflammatory reaction.

In the parts of the left coronary artery just beyond the aneurysm the intima was about five times as wide as the widest part of the media, the lumen of the vessel was extremely narrow, and the picture presented was largely one of repeated thrombosis, organization, and recanalization 
(Fig. 2). The internal elastic lamina proper and the muscle of the media showed the same degenerative changes as those in the wall of the aneurysm itself, but less advanced. The absence of inflammatory reaction was again a conspicuous feature. Further examination of the left coronary artery showed that, with progression to the periphery, there was a descending order of change.

Examination of the right coronary artery disclosed that, in this artery also, degenerative changes were present. The adventitia appeared normal, but there was extreme disorganization of the other elements of the arterial wall, and it was impossible to delimit the intima from the media with any exactitude (Fig. 3).

With regard to the vascular system outside the coronary tree, unfortunately no special parts of this were removed for histological examination, so that our examination related

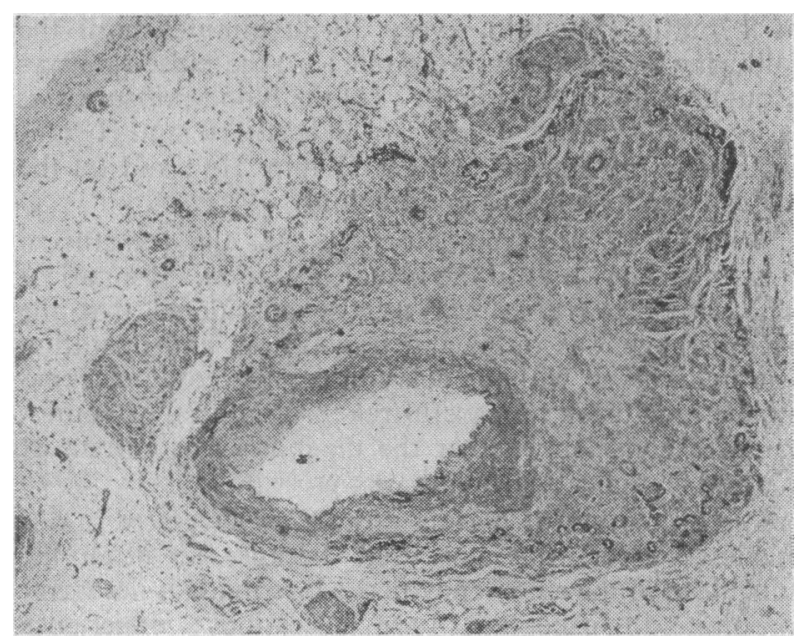

FIG. 3.-Right coronary artery at about $1.5 \mathrm{~cm}$. from right coronary ostium. (M.T. and V. $\times$ c. 48.$)$

only to that part of the aorta attached to the heart and to such vessels as happened to be in those parts of the other organs which were removed for histology. The small vessels in the substance of the liver, spleen, pancreas, kidneys, adrenals, lungs, and brain showed no histological abnormality. The aorta also was normal.

Examination of the heart muscle in both ventricles showed only various stages and types of change associated with ischaemia. In particular, there was no change suggestive of rheumatic carditis. The histological examination of the organs other than the heart merely confirmed the conclusion reached on naked-eye examination that the changes were the result of cardiac failure.

\section{Discussion}

The cause of the aneurysm of the left coronary artery is not clear. It is obvious, however, from the findings that the process which ultimately resulted in death had been going on for a much longer period than that of the terminal illness. The large healed scar in the interventricular septum betokens an extensive infarction at least 10 weeks prior to death. The aneurysm itself must have taken many weeks to assume its final appearance. On the other hand, this boy was described as a healthy active boy. He was well-developed physically, and we feel that the process which resulted in his death could not have been going on for more than a period of months. Although it is true to say that the cause is not clear, there are a number of findings which tend to eliminate some possible causes and to favour others.

Congenital Defect.-Incomplete embryonic development of the elastica and media in medium-sized arteries, especially at points of bifurcation, has been regarded as a possible cause of aneurysm formation since Forbus (1930) made a study of cerebral aneurysms. However, the muscle of the media and the elastica were, in this case, if anything, stronger at the junction of the two main branches of the left coronary artery than anywhere else at the site of the aneurysm. Scott (1948) believes that the assumption of a congenital origin is warranted in most cases in which there are other congenital cardiovascular anomalies. In this case there were none. .

Atherosclerosis.-Our opinion is that this can be dismissed as a possible cause of the aneurysm. There were no atheromatous plaques to be seen anywhere in the vascular system, and no evidence of lipid degeneration anywhere in the coronary tree.

Infectious Arteritis. - This cannot be ruled out as a cause of the aneurysm, but it must be admitted that there is no evidence of its nature or origin. There is no evidence of pericardial or myocardial infection. The picture is not that of a mycotic embolism. There is nothing to suggest bacterial endocarditis, nor do we think that syphilitic arteritis can be entertained.

Necrotizing Arteritis.-Rheumatic carditis, which has been known to cause necrotizing arteritis and coronary artery aneurysm (Rae, 1937), can, we think, be dismissed in this case. Neither the heart valves nor the myocardium showed any evidence of it. Polyarteritis nodosa, usually a systemic and diffuse disease, has been known to occur as a localized condition, and has been known to cause aneurysms of the coronary arteries (Spector, 1939 ; Sinclair and Nitsch, 1949). This diagnosis cannot be dismissed. The histological appearances are suggestive of a necrotic process affecting all the elements of the arterial wall, with the exception of the adventitia. It seems to us that the elastic tissue has suffered more severely than the other elements of the arterial wall. This is shown in the more peripheral sections of the arteries, where, although the muscle of the media has a normal appearance, the internal elastic lamina shows some degenerative changes. We are not aware of any destructive agent which singles out elastic tissue as its target, but we feel that degenerative changes in elastic tissue would repay closer study. Selective degeneration of the elastic tissue might explain some of the features of this case.

\section{Summary}

A case of sudden death in a boy of 9 was found to be due to localized aneurysm of the left coronary artery, resulting in thrombotic occlusion. Histological examination revealed that both coronary arteries were involved in a degenerative process affecting the intima and media of the arterial wall. The aetiology is discussed, but no definite conclusion is reached regarding the cause in this case.

We thank Professor D. H. Collins for his advice and helpful criticism in the preparation of this paper. Our thanks are also due to the late Mr. Norman S. Robson, H.M. Coroner for the County Borough of Rotherham, for permission to publish details of this case. We are indebted to Mr. S. J. Gray for the photographs.

\section{REFERENCES}

Crocker, D. W., Sobin, S., and Thomas, W. C. (1957). Amer. J. Path., 33, 819.

Forbus, W. D. (1930). Bull. Johns Hopk. Hosp. 47, 239.

Packard, M., and Wechsler, H. F. (1929). Arch. intern. Med., 43,

Rae, M. V. (1937). Arch. Path. (Chicago), 24, 369.

Scott, D. H. (1948) Amer. Heart J., 36, 403.

Sinclair, W., jun., and Nitsch, E (1949). Ibid., 38, 898.

Spector, S. (1939). Arch. Pediat., 56, 319. 